\title{
Stability Switches in a First-Order Complex Neutral Delay Equation
}

\author{
M. Roales and F. Rodríguez \\ Departamento de Matemática Aplicada, Universidad de Alicante, Apartado Postal 99, 03080 Alicante, Spain \\ Correspondence should be addressed to F. Rodríguez; f.rodriguez@ua.es
}

Received 23 July 2012; Accepted 12 November 2012

Academic Editor: Samir H. Saker

Copyright (C) 2013 M. Roales and F. Rodríguez. This is an open access article distributed under the Creative Commons Attribution License, which permits unrestricted use, distribution, and reproduction in any medium, provided the original work is properly cited.

Stability of the first-order neutral delay equation $x^{\prime}(t)+a x^{\prime}(t-\tau)=b x(t)+c x(t-\tau)$ with complex coefficients is studied, by analyzing the existence of stability switches.

\section{Introduction}

Delay differential equations (DDEs), and specifically DDEs of neutral type, appear in different scientific and technical problems, as in population dynamics, the modeling of networks containing lossless transmission lines, or the study of oscillations in elastic bars (see [1-3]).

The aim of this paper is to characterize the stability of the equation

$$
x^{\prime}(t)+a x^{\prime}(t-\tau)=b x(t)+c x(t-\tau),
$$

where $\tau>0$ is a constant delay and $a, b$, and $c$ are complex parameters.

In [4], Cahlon and Schmidt characterized the asymptotic behavior of the zero solution of the retarded equation with complex coefficients

$$
x^{\prime}(t)=p x(t)+q x(t-\tau),
$$

by transforming the complex equation into two coupled real DDEs. In [5], Wei and Zhang considered the same equation and, by studying the distribution of the roots of the characteristic equation for the associated real differential system with delay, analyzed the existence of stability switches [6-8].

Transforming a complex DDE into two coupled real DDEs to analyze its stability has some drawbacks, as, in general, the orders of the characteristic quasipolynomials to be analyzed double, and, since the study of the distribution of their roots is much more complicated as their degrees increase, it becomes very difficult to obtain necessary and sufficient conditions of stability $[1,9,10]$.

To avoid this problem, recently Li et al. [11] presented a method for directly analyzing the stability of complex DDEs on the basis of stability switches. Their results generalize those for real DDEs, thus greatly reducing the complexity of the analysis. In this paper, the results developed in [11] will be used to study the stability switches of the zero solution of the neutral equation (1).

\section{Previous Results}

For the sake of clarity in the exposition, we recall some results that will be used later, that may be found in [11].

Consider time-delay systems with a characteristic equation in the following form:

$$
\Delta(\lambda, \tau)=P(\lambda)+Q(\lambda) e^{-\lambda \tau}
$$

where $\tau \geq 0$ and $P(\lambda)$ and $Q(\lambda)$ are complex polynomial of order $n$ and $m$ respectively, with either $n>m$ or $n=m$ and $|\alpha|>|\beta|$, where $\alpha, \beta \in \mathbb{C}$ are the highest order coefficients of $P(\lambda)$ and $Q(\lambda)$, respectively. We will also assume that

$$
P(0)+Q(0) \neq 0,
$$

which states that $\lambda=0$ is not a root of (3), and that $P(\lambda)$ and $Q(\lambda)$ have no roots on the imaginary axis simultaneously. 
The assumed conditions are those required to use the result of $\mathrm{Li}$ et al. [11] stated below, but in other cases the stability of the system is mostly understood. Thus, if $n<m$, or $n=m$, and $|\alpha| \leq|\beta|$, there are clusterings of roots on a vertical line in the right half plane or the imaginary axis, and in the first of these cases the zero solution is unstable for all delays. Also, if (4) fails or $P(\lambda)$ and $Q(\lambda)$ have common roots on the imaginary axis, these common roots, or $\lambda=0$, are roots of $\Delta(\lambda, \tau)$ for all $\tau$, and for all delays the zero solution is not asymptotically stable.

Consider the function

$$
F(\omega)=|P(i \omega)|^{2}-|Q(i \omega)|^{2} .
$$

If $\omega^{*} \neq 0$ is a zero of $F(\omega)=0$, then there are an infinite number of delays $\tau_{j}$ corresponding to $\omega^{*}$ satisfying

$$
\Delta\left(i \omega^{*}, \tau_{j}\right)=0 .
$$

For these critical values, the following theorem [11, Theorem 1] characterizes the variation of the number of zeros with nonnegative real parts of $\Delta(\lambda, \tau)$, in terms of the order and sign of the first nonzero derivative of $F(\omega)$ evaluated at $\omega=$ $\omega^{*}$, extending to the complex coefficients setting previous results valid only for real DDEs $[8,12,13]$.

Theorem 1. Assume that $\Delta\left(i \omega^{*}, \tau_{j}\right)=0, j=0,1,2, \ldots$ Let $N(\tau)$ be the number of zeros with nonnegative real parts of $\Delta(\lambda, \tau)$, and let $M$ be an integer such that $F^{(M)}\left(\omega^{*}\right) \neq 0$, and $F^{(m)}\left(\omega^{*}\right)=0$ for all $m<M$. Then,

(a) $N(\tau)$ keeps unchanged as $\tau$ increases along $\tau_{j}$ if $M$ is even,

(b) when $M$ is odd, $N(\tau)$ increases by one if $\omega^{*} F^{(M)}\left(\omega^{*}\right)>$ 0 and decreases by one if $\omega^{*} F^{(M)}\left(\omega^{*}\right)<0$, as $\tau$ increases along $\tau_{j}$.

It should be noted that it is possible for a particular delay $\tau$ to produce more than one pure imaginary root, so that, in Theorem 1, the change in $N(\tau)$ refers to the change resulting from the specific critical value $\omega^{*}$.

\section{Stability Analysis of the First-Order Neutral DDE}

Consider the complex DDE (1), where

$$
a=a_{1}+i a_{2}, \quad b=b_{1}+i b_{2}, \quad c=c_{1}+i c_{2} .
$$

The characteristic equation associated with (1) is

$$
\lambda+a \lambda e^{-\lambda \tau}-b-c e^{-\lambda \tau}=0,
$$

so that

$$
\Delta(\lambda, \tau)=P(\lambda)+Q(\lambda) e^{-\lambda \tau}
$$

where

$$
P(\lambda)=\lambda-b, \quad Q(\lambda)=a \lambda-c .
$$

Since the order of both polynomials is 1 , we must demand that

$$
|a|<1 \text {. }
$$

As pointed out before, if $|a| \geq 1$ there are clusterings of roots on a vertical line in the right half plane, when $|a|>1$, in which case the system is unstable for all delays, or on the imaginary axis, when $|a|=1$. In this last critical case, the stability of the systems is not so clear, and a detailed, different type of analysis is required, as carried out in [8, pages 70-72] in the much simpler setting of real coefficients.

The following lemma gives $N(0)$, the number of zeros with nonnegative real parts of $\Delta(\lambda, \tau)$ when the delay is zero.

Lemma 2. If

$$
\left(b_{1}+c_{1}\right)\left(1+a_{1}\right)+\left(b_{2}+c_{2}\right) a_{2}<0,
$$

then $N(0)=0$. Otherwise, $N(0)=1$.

Proof. Consider the equation

$$
\Delta(\lambda, 0)=P(\lambda)+Q(\lambda)=\lambda-b+a \lambda-c=0 .
$$

Then

$$
\begin{aligned}
\lambda= & \frac{b+c}{1+a}=\frac{\left(b_{1}+c_{1}\right)+i\left(b_{2}+c_{2}\right)}{1+a_{1}+i a_{2}} \\
= & \left(\left[\left(b_{1}+c_{1}\right)\left(1+a_{1}\right)+\left(b_{2}+c_{2}\right) a_{2}\right]\right. \\
& \left.+i\left[\left(b_{2}+c_{2}\right)\left(1+a_{1}\right)-\left(b_{1}+c_{1}\right) a_{2}\right]\right) \\
& \times\left(\left(1+a_{1}\right)^{2}+a_{2}^{2}\right)^{-1},
\end{aligned}
$$

and therefore

$$
\mathfrak{R}(\lambda)<0 \Longleftrightarrow\left(b_{1}+c_{1}\right)\left(1+a_{1}\right)+\left(b_{2}+c_{2}\right) a_{2}<0,
$$

and thus $N(0)=0$. If $\Re(\lambda) \geq 0$, there is only one root with real part nonnegative, and hence $N(0)=1$.

Now consider the function

$$
\begin{aligned}
F(\omega)= & |P(i \omega)|^{2}-|Q(i \omega)|^{2} \\
= & \left(\omega-b_{2}\right)^{2}+b_{1}^{2}-\left(a_{1} \omega-c_{2}\right)^{2}-\left(a_{2} \omega+c_{1}\right)^{2} \\
= & \omega^{2}\left(1-a_{1}^{2}-a_{2}^{2}\right)+\omega\left(2 a_{1} c_{2}-2 b_{2}-2 a_{2} c_{1}\right) \\
& +b_{1}^{2}+b_{2}^{2}-c_{1}^{2}-c_{2}^{2} \\
= & \omega^{2}\left(1-|a|^{2}\right)-2 \mathfrak{J}(b+a \bar{c}) \omega+|b|^{2}-|c|^{2},
\end{aligned}
$$

and calculate its zeros. One gets

$$
\omega=\frac{\mathfrak{J}(b+a \bar{c}) \pm B}{1-|a|^{2}},
$$

where

$$
B=\sqrt{(\mathfrak{I}(b+a \bar{c}))^{2}-\left(1-|a|^{2}\right)\left(|b|^{2}-|c|^{2}\right)} .
$$


First, we assume that

$$
\mathfrak{J}(b+a \bar{c})>0,
$$

and consider several subcases.

If $(\Im(b+a \bar{c}))^{2}-\left(1-|a|^{2}\right)\left(|b|^{2}-|c|^{2}\right)<0$, then $F(\omega)$ has no real root, and therefore the stability of the zero solution of

(1) does not change for any $\tau>0$.

If $(\Im(b+a \bar{c}))^{2}-\left(1-|a|^{2}\right)\left(|b|^{2}-|c|^{2}\right)>0$, then $F(\omega)$ has two real roots, $\omega_{+}$, and $\omega_{-}$, such that

$$
\begin{aligned}
& |b|^{2}-|c|^{2}>0 \Longrightarrow \omega_{+}>\omega_{-}>0, \\
& |b|^{2}-|c|^{2}<0 \Longrightarrow \omega_{+}>0>\omega_{-}, \\
& |b|^{2}-|c|^{2}=0 \Longrightarrow \omega_{+}>\omega_{-}=0,
\end{aligned}
$$

this last possibility being excluded from the analysis, since $\lambda=0$ is a root of (3), a contradiction to (4).

Consider the case where $\omega_{+}>\omega_{-}>0$. Substituting $\lambda=i \omega$ into (8), and separating the real and imaginary parts, one gets

$$
\begin{gathered}
\omega-b_{2}+\left(a_{2} \omega+c_{1}\right) \sin \omega \tau+\left(a_{1} \omega-c_{2}\right) \cos \omega \tau=0, \\
-b_{1}+\left(a_{1} \omega-c_{2}\right) \sin \omega \tau-\left(a_{2} \omega+c_{1}\right) \cos \omega \tau=0,
\end{gathered}
$$

obtaining the following two sets of values of $\tau$ for which there are roots,

$$
\tau_{n, 1}=\frac{\theta_{1}}{\omega_{+}}+\frac{2 n \pi}{\omega_{+}}, \quad n=0,1,2, \ldots,
$$

where $0 \leq \theta_{1}<2 \pi$ and

$$
\begin{gathered}
\cos \theta_{1}=\frac{\left(b_{2}-\omega_{+}\right)\left(a_{1} \omega_{+}-c_{2}\right)-b_{1}\left(a_{2} \omega_{+}+c_{1}\right)}{\left(a_{1} \omega_{+}-c_{2}\right)^{2}+\left(a_{2} \omega_{+}+c_{1}\right)^{2}}, \\
\sin \theta_{1}=\frac{\left(b_{2}-\omega_{+}\right)\left(a_{2} \omega_{+}+c_{1}\right)+b_{1}\left(a_{1} \omega_{+}-c_{2}\right)}{\left(a_{1} \omega_{+}-c_{2}\right)^{2}+\left(a_{2} \omega_{+}+c_{1}\right)^{2}}, \\
\tau_{n, 2}=\frac{\theta_{2}}{\omega_{-}}+\frac{2 n \pi}{\omega_{-}}, \quad n=0,1,2, \ldots,
\end{gathered}
$$

where $0 \leq \theta_{2}<2 \pi$ and

$$
\begin{aligned}
& \cos \theta_{2}=\frac{\left(b_{2}-\omega_{-}\right)\left(a_{1} \omega_{+}-c_{2}\right)-b_{1}\left(a_{2} \omega_{-}+c_{1}\right)}{\left(a_{1} \omega_{-}-c_{2}\right)^{2}+\left(a_{2} \omega_{-}+c_{1}\right)^{2}}, \\
& \sin \theta_{2}=\frac{\left(b_{2}-\omega_{-}\right)\left(a_{2} \omega_{-}+c_{1}\right)+b_{1}\left(a_{1} \omega_{-}-c_{2}\right)}{\left(a_{1} \omega_{-}-c_{2}\right)^{2}+\left(a_{2} \omega_{-}+c_{1}\right)^{2}} .
\end{aligned}
$$

Since

$$
F^{\prime}\left(\omega_{+}\right)=-F^{\prime}\left(\omega_{-}\right)=B>0,
$$

one has

$$
\begin{aligned}
& \omega_{+} F^{\prime}\left(\omega_{+}\right)>0, \\
& \omega_{-} F^{\prime}\left(\omega_{-}\right)<0 .
\end{aligned}
$$

Therefore, according to Theorem 1, the number of the characteristic roots with nonnegative real parts increases by one as $\tau$ passes through $\tau_{n, 1}$ and decreases by one as $\tau$ passes through $\tau_{n, 2}$.

If $N(0)=0$, that is, if the zero solution of (1) is stable for $\tau=0$, then it must follow that $\tau_{0,1}<\tau_{0,2}$, since $N(\tau)$ cannot become negative. There are stability switches when the delays are such that

$$
\tau_{0,1}<\tau_{0,2}<\tau_{1,1}<\tau_{1,2}<\ldots
$$

Since

$$
\tau_{n+1,1}-\tau_{n, 1}=\frac{2 \pi}{\omega_{+}}<\frac{2 \pi}{\omega_{-}}=\tau_{n+1,2}-\tau_{n, 2},
$$

the intervals become smaller with increasing $n$, so that eventually, for a certain $k \geq 1$,

$$
\tau_{k-1,1}<\tau_{k, 1} \leq \tau_{k-1,2}
$$

Thus, the distribution of delays is

$$
\tau_{0,1}<\tau_{0,2}<\cdots<\tau_{k-1,1}<\tau_{k, 1} \leq \tau_{k-1,2}<\tau_{k+1,1}<\cdots
$$

and there is only a finite number of stability switches, with the system becoming unstable for $\tau>\tau_{k-1,1}$. Under these conditions, if $N(0)=1$, that is, if the zero solution of (1) is unstable for $\tau=0$, the system cannot be stabilized.

If $N(0)=1$, stability switches occur when the distribution of delays is

$$
\tau_{0,2}<\tau_{0,1}<\cdots<\tau_{k-1,2}<\tau_{k-1,1}<\tau_{k, 1} \leq \tau_{k, 2}<\tau_{k+1,1}<\cdots,
$$

in which case there are $k$ switches from instability to stability to instability. Once $\tau_{k, 1} \leq \tau_{k, 2}$, stability switch stops, so that the system becomes unstable for $\tau>\tau_{k-1,1}$.

The conditions on the delays for the above orderings to be valid can be formulated directly from (22) to (25).

Now we study the case when $\omega_{+}>0>\omega_{-}$. Proceeding as before, there are two sets of critical values of delays, $\tau_{n, 1}$ and $\tau_{n, 2}$, corresponding to $\omega_{+}$and $\omega_{-}$, respectively, but now it holds that $\omega_{+} F^{\prime}\left(\omega_{+}\right)$and $\omega_{-} F^{\prime}\left(\omega_{-}\right)$are both positive. Hence, the number of the characteristic roots with nonnegative real parts increases by one as $\tau$ passes through $\tau_{n, 1}$ or $\tau_{n, 2}$. If $N(0)=0$, then there is $\tau_{0,1}$ such that the zero solution of (1) is stable for $\tau<\tau_{0,1}$ and it becomes unstable for $\tau>\tau_{0,1}$. If $N(0)=1$, the zero solution of (1) never becomes stable for any $\tau>0$.

To finish the analysis when $\mathfrak{\Im}(b+a \bar{c})>0$, let

$$
(\mathfrak{I}(b+a \bar{c}))^{2}-\left(1-|a|^{2}\right)\left(|b|^{2}-|c|^{2}\right)=0,
$$

so that $F(\omega)$ has a repeated real root,

$$
\omega^{*}=\frac{2 \mathfrak{J}(b+a \bar{c})}{1-|a|^{2}}>0 .
$$


For this root, as in the previous cases, there is a set of critical delays $\tau_{n}$. Since $F^{\prime}\left(\omega^{*}\right)=0$, we have to consider the second derivative,

$$
F^{\prime \prime}\left(\omega^{*}\right)=2\left(1-|a|^{2}\right) \neq 0 .
$$

According to Theorem 1, since $M=2$ is even, $N(\tau)$ keeps unchanged as $\tau$ increases along $\tau_{n}$. We conclude that the stability of the zero solution of (1) does not change for any $\tau>0$.

Finally, consider the case when

$$
\mathfrak{J}(b+a \bar{c})<0 .
$$

The analysis and results of this case are much similar to the previous one, and we will omit the details. There are only minor differences when

$$
(\mathfrak{I}(b+a \bar{c}))^{2}-\left(1-|a|^{2}\right)\left(|b|^{2}-|c|^{2}\right)>0,
$$

and $|b|^{2}-|c|^{2}>0$.

In this subcase, the function $F(\omega)$ has two roots, $0>\omega_{+}>$ $\omega_{-}$, with corresponding sets of critical delays,

$$
\tau_{n, 1}=\frac{\theta_{1}}{\omega_{+}}-\frac{2 n \pi}{\omega_{+}}, \quad \tau_{n, 2}=\frac{\theta_{2}}{\omega_{-}}-\frac{2 n \pi}{\omega_{-}}, \quad n=0,1,2, \ldots,
$$

with $-2 \pi \leq \theta_{1}<0$ and $-2 \pi \leq \theta_{2}<0$ satisfying the expressions (23) and (25), respectively. It holds that $\omega_{+} F^{\prime}\left(\omega_{+}\right)<0$ and $\omega_{-} F^{\prime}\left(\omega_{-}\right)>0$, and hence, from Theorem 1 , the number of the characteristic roots with nonnegative real parts decreases by one as $\tau$ passes through $\tau_{n, 1}$ and increases by one as $\tau$ passes through $\tau_{n, 2}$. Also, since

$$
\tau_{n+1,2}-\tau_{n, 2}=\frac{-2 \pi}{\omega_{-}}<\frac{-2 \pi}{\omega_{+}}=\tau_{n+1,1}-\tau_{n, 1}
$$

only a finite number of stability switches may exist.

If $N(0)=0$, then it must follow that $\tau_{0,2}<\tau_{0,1}$, and there are $k$ switches from stability to instability when the distribution of the critical delays is

$$
\tau_{0,2}<\tau_{0,1}<\cdots<\tau_{k-1,2}<\tau_{k, 2} \leq \tau_{k-1,1}<\tau_{k+1,2}<\ldots
$$

Once $\tau_{k, 2} \leq \tau_{k-1,1}$, stability switch stops and the system becomes unstable for $\tau>\tau_{k-1,2}$.

Similarly, if $N(0)=1$, stability switches may occur for the distribution of critical delays

$$
\tau_{0,1}<\tau_{0,2}<\cdots<\tau_{k-1,1}<\tau_{k-1,2}<\tau_{k, 2} \leq \tau_{k, 1}<\tau_{k+1,2} \cdots
$$

Once $\tau_{k, 2} \leq \tau_{k, 1}$, stability switch stops and the system remains unstable.

In summary, the following theorem has been established.

Theorem 3. Under the hypotheses of Theorem 1, consider the first-order complex neutral delay equation (1), where a satisfies condition (11).

(a) If $\mathfrak{I}(b+a \bar{c}) \neq 0$, and $(\mathfrak{J}(b+a \bar{c}))^{2}-\left(1-|a|^{2}\right)\left(|b|^{2}-\right.$ $\left.|c|^{2}\right) \leq 0$
(1) if $N(0)=0$, then the zero solution of (1) is stable for all $\tau \geq 0$;

(2) if $N(0)=1$, then the zero solution of (1) is unstable for all $\tau \geq 0$.

(b) If $\mathfrak{J}(b+a \bar{c}) \neq 0,(\mathfrak{J}(b+a \bar{c}))^{2}-\left(1-|a|^{2}\right)\left(|b|^{2}-|c|^{2}\right)>0$, and $|b|^{2}-|c|^{2}<0$,

(1) if $N(0)=0$, then there is $\tau_{0,1}$ such that the zero solution of (1) is stable for $\tau<\tau_{0,1}$ and it becomes unstable for $\tau>\tau_{0,1}$;

(2) if $N(0)=1$, the zero solution of (1) is always unstable.

(c) If $\mathfrak{J}(b+a \bar{c})>0,(\mathfrak{I}(b+a \bar{c}))^{2}-\left(1-|a|^{2}\right)\left(|b|^{2}-|c|^{2}\right)>0$, and $|b|^{2}-|c|^{2}>0$

(1) if $N(0)=0$ and the distribution of critical delays is

$\tau_{0,1}<\tau_{0,2}<\cdots<\tau_{k-1,1}<\tau_{k, 1} \leq \tau_{k-1,2}<\tau_{k+1,1}<\cdots$,

then the zero solution of (1) is asymptotically stable for $\tau<\tau_{0,1}$ and $\tau \in \bigcup_{j=0}^{k-2}\left(\tau_{j, 2}, \tau_{j+1,1}\right)$ and unstable for $\tau \in \bigcup_{j=0}^{k-2}\left(\tau_{j, 1}, \tau_{j, 2}\right)$ and $\tau>\tau_{k-1,1}$;

(2) if $N(0)=1$ and the distribution of critical delays is

$\tau_{0,2}<\tau_{0,1}<\cdots<\tau_{k-1,2}<\tau_{k-1,1}<\tau_{k, 1} \leq \tau_{k, 2}<\tau_{k+1,1}<\cdots$,

then the zero solution of (1) is asymptotically stable for $\tau \in \bigcup_{j=0}^{k-1}\left(\tau_{j, 2}, \tau_{j, 1}\right)$ and unstable for $\tau<\tau_{0,2}, \tau \in \bigcup_{j=0}^{k-2}\left(\tau_{j, 1}, \tau_{j+1,2}\right)$, and $\tau>\tau_{k-1,1}$.

(d) If $\mathfrak{\Im}(b+a \bar{c})<0,(\mathfrak{I}(b+a \bar{c}))^{2}-\left(1-|a|^{2}\right)\left(|b|^{2}-|c|^{2}\right)>0$, and $|b|^{2}-|c|^{2}>0$,

(1) if $N(0)=0$ and the distribution of critical delays is

$\tau_{0,2}<\tau_{0,1}<\cdots<\tau_{k-1,2}<\tau_{k, 2} \leq \tau_{k-1,1}<\tau_{k+1,2}<\cdots$,

then the zero solution of (1) is asymptotically stable for $\tau<\tau_{0,2}$ and $\tau \in \bigcup_{j=0}^{k-2}\left(\tau_{j, 1}, \tau_{j+1,2}\right)$, and unstable for $\tau \in \bigcup_{j=0}^{k-2}\left(\tau_{j, 2}, \tau_{j, 1}\right)$ and $\tau>\tau_{k-1,2}$;

(2) if $N(0)=1$ and the distribution of critical delays is

$\tau_{0,1}<\tau_{0,2}<\cdots<\tau_{k-1,1}<\tau_{k-1,2}<\tau_{k, 2} \leq \tau_{k, 1}<\tau_{k+1,2} \cdots$,

then the zero solution of (1) is asymptotically stable for $\tau \in \bigcup_{j=0}^{k-1}\left(\tau_{j, 1}, \tau_{j, 2}\right)$ and unstable for $\tau<\tau_{0,1}, \tau \in \bigcup_{j=0}^{k-2}\left(\tau_{j, 2}, \tau_{j+1,1}\right)$, and $\tau>\tau_{k-1,2}$. 
Finally, we will consider the case

$$
\mathfrak{J}(b+a \bar{c})=0
$$

for which

$$
F(\omega)=\omega^{2}\left(1-|a|^{2}\right)+|b|^{2}-|c|^{2} .
$$

If $|b|^{2}-|c|^{2}>0$, then $F(\omega)$ has no real root, and therefore the stability of the zero solution of (1) does not change for any $\tau \geq 0$.

If $|b|^{2}-|c|^{2}<0$, then $F(\omega)$ has two real roots,

$$
\omega_{+}=\sqrt{\frac{|c|^{2}-|b|^{2}}{1-|a|^{2}}}, \quad \omega_{-}=-\sqrt{\frac{|c|^{2}-|b|^{2}}{1-|a|^{2}}}
$$

with corresponding sets of critical delays

$$
\tau_{n, 1}=\frac{\theta_{1}}{\omega_{+}}+\frac{2 n \pi}{\omega_{+}}, \quad n=0,1,2, \ldots
$$

where $0 \leq \theta_{1}<2 \pi$, and

$$
\begin{aligned}
& \cos \theta_{1}=\left(\left(b_{2}-\sqrt{\left(|c|^{2}-|b|^{2}\right) /\left(1-|a|^{2}\right)}\right)\right. \\
& \left.\times\left(a_{1} \sqrt{\left(|c|^{2}-|b|^{2}\right) /\left(1-|a|^{2}\right)}-c_{2}\right)\right) \\
& \times\left(b_{1}^{2}+\left(\sqrt{\left(|c|^{2}-|b|^{2}\right) /\left(1-|a|^{2}\right)}-b_{2}\right)^{2}\right)^{-1} \\
& -\left(b_{1}\left(a_{2} \sqrt{\left(|c|^{2}-|b|^{2}\right) /\left(1-|a|^{2}\right)}+c_{1}\right)\right) \\
& \times\left(b_{1}^{2}+\left(\sqrt{\left(|c|^{2}-|b|^{2}\right) /\left(1-|a|^{2}\right)}-b_{2}\right)^{2}\right)^{-1}, \\
& \sin \theta_{1}=\left(\left(b_{2}-\sqrt{\left(|c|^{2}-|b|^{2}\right) /\left(1-|a|^{2}\right)}\right)\right. \\
& \left.\times\left(a_{2} \sqrt{\left(|c|^{2}-|b|^{2}\right) /\left(1-|a|^{2}\right)}+c_{1}\right)\right) \\
& \times\left(b_{1}^{2}+\left(\sqrt{\left(|c|^{2}-|b|^{2}\right) /\left(1-|a|^{2}\right)}-b_{2}\right)^{2}\right)^{-1} \\
& +\left(b_{1}\left(a_{1} \sqrt{\left(|c|^{2}-|b|^{2}\right) /\left(1-|a|^{2}\right)}-c_{2}\right)\right) \\
& \times\left(b_{1}^{2}+\left(\sqrt{\left(|c|^{2}-|b|^{2}\right) /\left(1-|a|^{2}\right)}-b_{2}\right)^{2}\right)^{-1}, \\
& \tau_{n, 2}=\frac{\theta_{2}}{\omega_{-}}-\frac{2 n \pi}{\omega_{-}}, \quad n=0,1,2, \ldots,
\end{aligned}
$$

where $-2 \pi \leq \theta_{2}<0$, and

$$
\begin{aligned}
\cos \theta_{2}= & \left(\left(b_{2}+\sqrt{\left(|c|^{2}-|b|^{2}\right) /\left(1-|a|^{2}\right)}\right)\right. \\
& \left.\times\left(-a_{1} \sqrt{\left(|c|^{2}-|b|^{2}\right) /\left(1-|a|^{2}\right)}-c_{2}\right)\right) \\
& \times\left(b_{1}^{2}+\left(\sqrt{\left(|c|^{2}-|b|^{2}\right) /\left(1-|a|^{2}\right)}+b_{2}\right)^{2}\right)^{-1} \\
& -\left(b_{1}\left(-a_{2} \sqrt{\left(|c|^{2}-|b|^{2}\right) /\left(1-|a|^{2}\right)}+c_{1}\right)\right) \\
& \times\left(b_{1}^{2}+\left(\sqrt{\left(|c|^{2}-|b|^{2}\right) /\left(1-|a|^{2}\right)}+b_{2}\right)^{2}\right)^{-1}, \\
\sin \theta_{2}= & \left(\left(b_{2}+\sqrt{\left(|c|^{2}-|b|^{2}\right) /\left(1-|a|^{2}\right)}\right)\right. \\
& \left.\times\left(-a_{2} \sqrt{\left(|c|^{2}-|b|^{2}\right) /\left(1-|a|^{2}\right)}+c_{1}\right)\right)^{2} \\
& \times\left(b_{1}^{2}+\left(\sqrt{\left(|c|^{2}-|b|^{2}\right) /\left(1-|a|^{2}\right)}+b_{2}\right)^{2}\right)^{-1} \\
& +\left(b_{1}\left(-a_{1} \sqrt{\left(|c|^{2}-|b|^{2}\right) /\left(1-|a|^{2}\right)}-c_{2}\right)\right) \\
& \left.+\left(\sqrt{\left(|c|^{2}-|b|^{2}\right) /\left(1-|a|^{2}\right)}+b_{2}\right)^{2}\right)^{-1} .
\end{aligned}
$$

In this case, it holds that $\omega_{+} F^{\prime}\left(\omega_{+}\right)$and $\omega_{-} F^{\prime}\left(\omega_{-}\right)$are both positive, and hence, from Theorem 1 , the number of the characteristic roots with nonnegative real parts increases by one as $\tau$ passes either through $\tau_{n, 1}$ or $\tau_{n, 2}$. Thus, the following theorem has been established.

Theorem 4. Under the hypotheses of Theorem 1, consider (1) satisfying condition (11). If $\mathfrak{\Im}(b+a \bar{c})=0$,

(a) if $|b|^{2}-|c|^{2}>0$, then the zero solution of (1) is always stable if $N(0)=0$ and unstable if $N(0)=1$;

(b) if $|b|^{2}-|c|^{2}<0$, if $N(0)=0$ then there is $\tau_{0,1}$ such that the zero solution of (1) is stable for $\tau<\tau_{0,1}$ and it becomes unstable for $\tau>\tau_{0,1}$. If $N(0)=1$, the zero solution of (1) never becomes stable for any $\tau \geq 0$.

\section{Acknowledgments}

The authors wish to thank the reviewers for useful comments and suggestions, which improved the paper. F. Rodríguez is grateful for financial support from project FEEDBACK (CGL2011-30515-C02-01), funded by the Spanish Ministry of Science and Innovation.

\section{References}

[1] K. Gopalsamy, Stability and Oscillations in Delay Differential Equations of Population Dynamics, Kluwer, Dordrecht, The Netherlands, 1992. 
[2] R. D. Driver, "A mixed neutral system," Nonlinear Analysis: Theory, Methods and Applications A, vol. 8, no. 2, pp. 155-158, 1984.

[3] R. K. Brayton and R. A. Willoughby, "On the numerical integration of a symmetric system of difference-differential equations of neutral type," Journal of Mathematical Analysis and Applications, vol. 18, pp. 182-189, 1967.

[4] B. Cahlon and D. Schmidt, "On stability of a first-order complex delay differential equation," Nonlinear Analysis: Real World Applications, vol. 3, no. 3, pp. 413-429, 2002.

[5] J. Wei and C. Zhang, "Stability analysis in a first-order complex differential equations with delay," Nonlinear Analysis: Theory, Methods and Applications A, vol. 59, no. 5, pp. 657-671, 2004.

[6] K. L. Cooke and Z. Grossman, "Discrete delay, distributed delay and stability switches," Journal of Mathematical Analysis and Applications, vol. 86, no. 2, pp. 592-627, 1982.

[7] E. Beretta and Y. Kuang, "Geometric stability switch criteria in delay differential systems with delay dependent parameters," SIAM Journal on Mathematical Analysis, vol. 33, no. 5, pp. 11441165, 2002.

[8] Y. Kuang, Delay Differential Equations with Applications in Population Dynamics, Academic Press, Arizona, Ariz, USA, 1993.

[9] S. Ruan and J. Wei, "On the zeros of a third degree exponential polynomial with applications to a delayed model for the control of testosterone secretion," IMA Journal of Mathematics Applied in Medicine and Biology, vol. 18, pp. 41-52, 2001.

[10] X. Li and J. Wei, "On the zeros of a fourth degree exponential polynomial with applications to a neural network model with delays," Chaos, Solitons and Fractals, vol. 26, no. 2, pp. 519-526, 2005.

[11] J. Li, L. Zhang, and Z. Wang, "Two effective stability criteria for linear time-delay systems with complex coefficients," Journal of Systems Science and Complexity, vol. 24, no. 5, pp. 835-849, 2011.

[12] M. S. Lee and C. S. Hsu, "On the $\tau$-decomposition method of stability analysis for retarded dynamical systems," SIAM Journal on Control and Optimization, vol. 7, pp. 215-233, 1969.

[13] K. L. Cooke and P. van den Driessche, "On zeroes of some transcendental equations," Funkcialaj Ekvacioj, vol. 29, no. 1, pp. 77-90, 1986. 


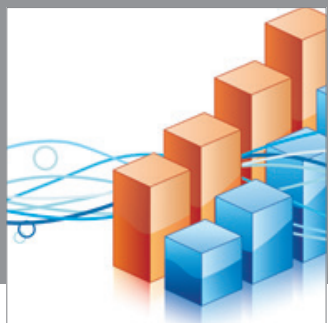

Advances in

Operations Research

mansans

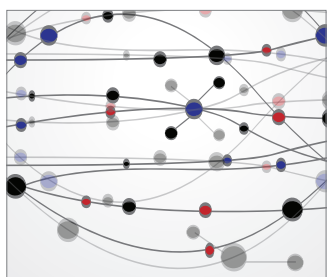

The Scientific World Journal
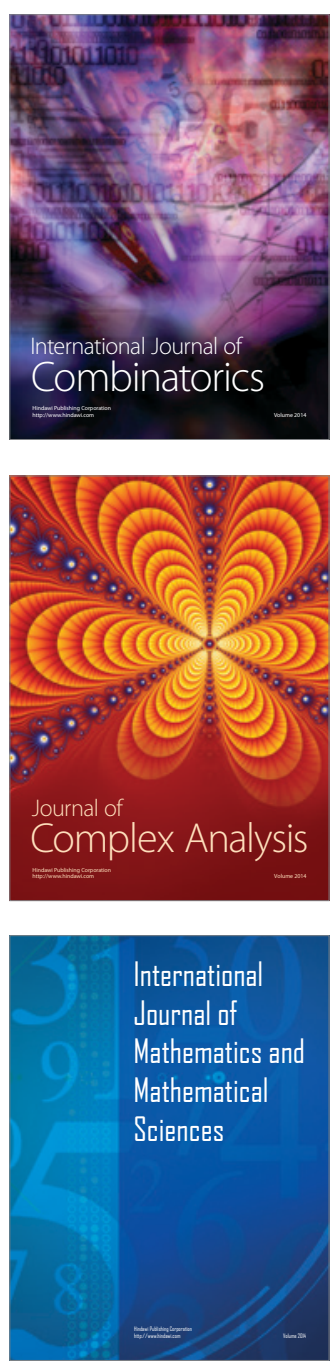
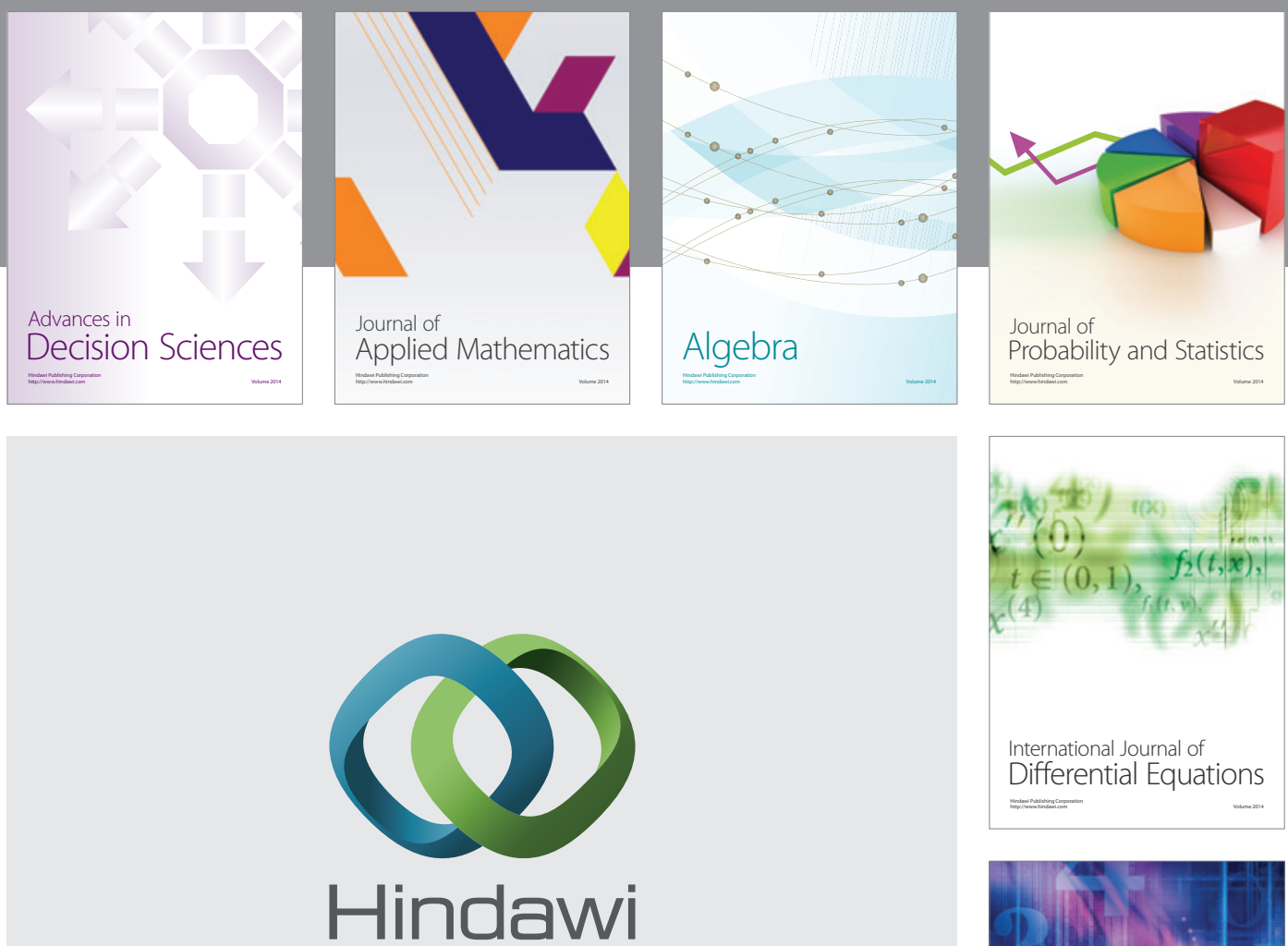

Submit your manuscripts at http://www.hindawi.com
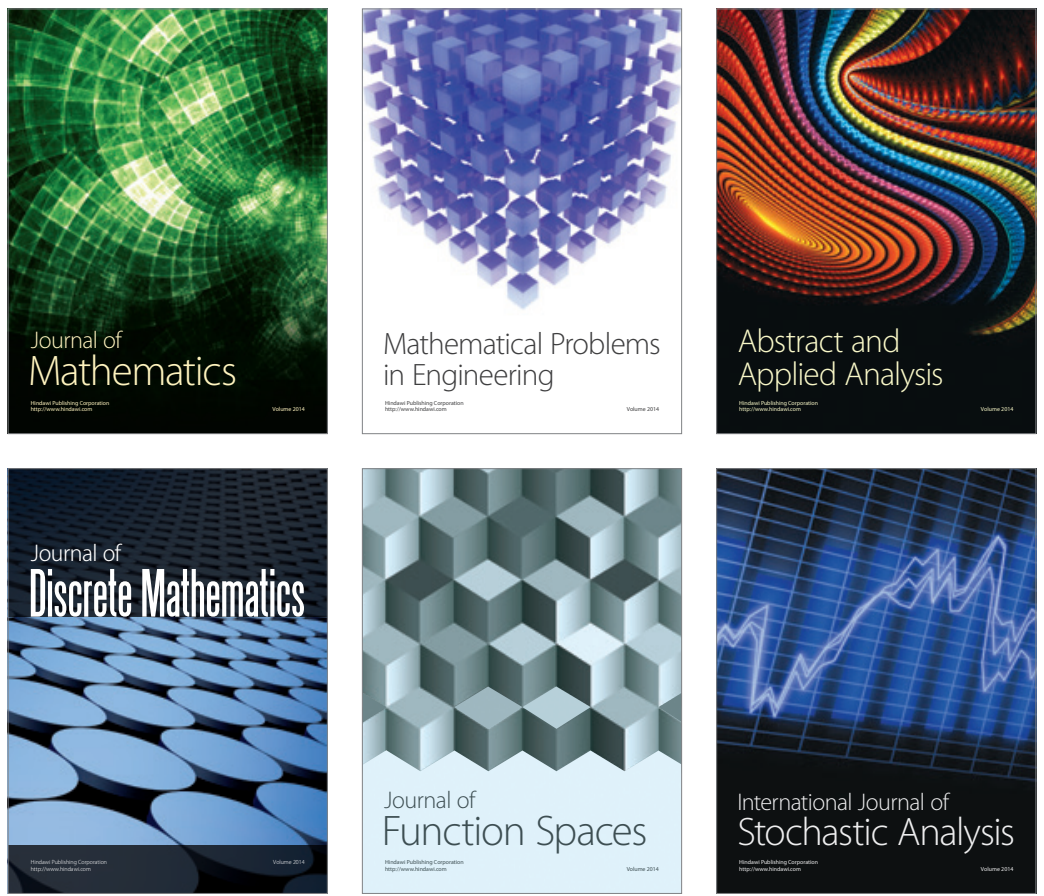

Journal of

Function Spaces

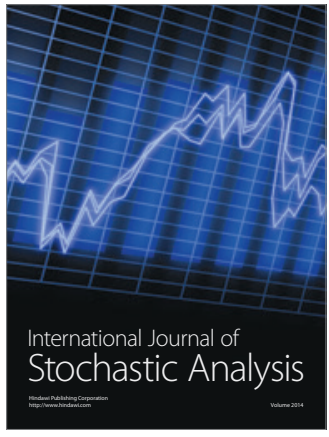

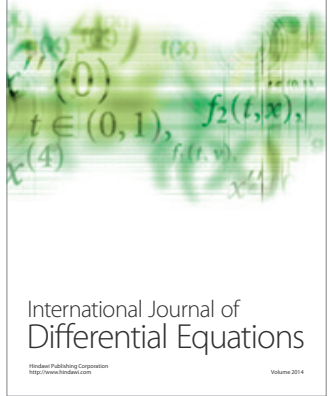
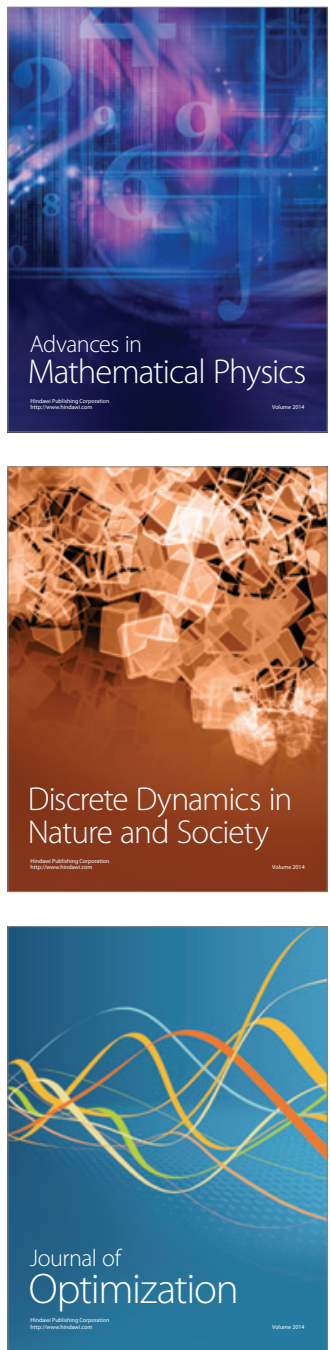